\title{
Velocity-based Leader-follower Trajectory Tracking for Nonholonomic Mobile Robots using Recurrent Fuzzy Neural Network with Noisy Measurements
}

\author{
Samira Eshghi ${ }^{1}$, Jinjun Shan ${ }^{1}$ \\ ${ }^{1}$ Department of Earth and Space Science and Engineering, York University, 4700 Keele Street, Toronto M3J1P3, Canada \\ *seshghi@yorku.ca
}

\begin{abstract}
This paper proposes a distributed control architecture for cooperative control of multiple nonholonomic mobile robots subject to uncertain dynamics and noisy measurements. Collaboration of multiple mobile robots for transportation, exploration, search and rescue and many other tasks is in high demand for research and industrial applications. The fleet of robots communicate with each other via communication network, hence network-induced noises are to be expected during operation. In addition, the motion of the robot is often described only by kinematics, and the robot dynamic is normally ignored. This may lead to some performance adversities such as jerk in tracking trajectories with sharp turns or in fast maneuvers. These uncertainties can cause collision between agents or failure in accomplishing collaborative tasks in challenging environments. Hence, a Recurrent Fuzzy Neural Network is designed to approximate unknown nonlinear functions due to unmodeled dynamics and uncertainties. A leader-follower system is modeled to track a desired velocity profile based on a desired trajectory. The proposed control architecture is simulated to verify the robustness of the system in presence of communication delays and unmodeled dynamics.
\end{abstract}

Word count: 240 